\title{
Matching Dominating Sets of Direct Product Graphs of Cayley Graphs with Arithmetic Graphs
}

\author{
S. Uma Maheswari \\ Lecturer \\ Department of Mathematics \\ JMJ College For Women \\ Tenali, AP, India
}

\author{
B. Maheswari \\ Professor \\ Department of \\ Applied Mathematics \\ S.P. Women's University \\ Tirupati, AP, India
}

\author{
M. Manjuri \\ Department of \\ Applied Mathematics \\ S.P. Women's University \\ Tirupati, AP, India
}

\begin{abstract}
Graph Theory is one of the most flourishing branches of modern Mathematics finding widest applications in all most all branches of Science \& Technology. It is applied in diverse areas such as social sciences, linguistics, physical sciences, communication engineering etc. Number Theory is one of the oldest branches of Mathematics, which inherited rich contributions from almost all greatest mathematicians, ancient and modern.
\end{abstract}

Every branch of mathematics employs some notion of a product that enables the combination or decomposition of its elemental structures. Product of graphs are introduced in Graph Theory very recently and developing rapidly.

In this paper, we consider direct product graphs of Cayley graphs with Arithmetic graphs and present Matching dominating set of these graphs.

\section{Keywords}

Euler totient Cayley Graph, Arithmetic $V_{n}$ graph, Direct Product Graph, Matching dominating set.

AMS (MOS) Subject Classification: 6905c

\section{INTRODUCTION}

\subsection{Euler Totient Cayley Graph $G\left(Z_{n}, \varphi\right)$}

Madhavi [1] introduced the concept of Euler totient Cayley graphs and studied some of its properties.

For any positive integer $n \geq 1$, the number of positive integers less than $n$ and relatively prime to $n$ is denoted by $\varphi(n)$ and is called Euler totient function. Let $S$ denote the set of all positive integers less than $n$ and relatively prime to $n$. That is $S=\{r / 1 \leq r<n$ and $\operatorname{GCD}(r, n)=1\}$. Then $|S|=\varphi(n)$.

Now we define Euler totient Cayley graph as follows.

For each positive integer $n$, let $Z_{n}$ be the additive group of integers modulo $n$ and let $S$ be the set of all integers less than $n$ and relatively prime to $n$. The Euler totient Cayley graph $G\left(Z_{n}, \varphi\right)$ is defined as the graph whose vertex set $V$ is given by $Z_{n}=\{0,1,2, \ldots . n-1\}$ and the edge set is $E=\{(x, y) / x-y \in S$ or $y-x \in S\}$.
Clearly as proved by Madhavi [1], the Euler Totient Cayley graph $G\left(Z_{n}, \varphi\right)$ is

1. a connected, simple and undirected graph,

2. $\Phi(n)$ - regular and has $n . \Phi(n) / 2$ edges,

3. Hamiltonian,

4. Eulerian for $n \geq 3$,

5. Bipartite if $n$ is even,

6. Complete graph if $n$ is a prime.

\subsection{Arithmetic $V_{n}$ Graph}

Vasumathi [2] introduced the concept of Arithmetic $V_{n}$ graphs and studied some of its properties. Their definition of Arithmetic $V_{n}$ graph is as follows.

Let $n$ be a positive integer such that $n=p_{1}^{\alpha_{1}} p_{2}^{\alpha_{2}} \ldots \ldots . . p_{k}^{\alpha_{k}}$. Then the Arithmetic $V_{n}$ graph is defined as the graph whose vertex set consists of the divisors of $n$ and two vertices $u, v$ are adjacent in $V_{n}$ graph if and only if $\operatorname{GCD}(u, v)=p_{i}$, for some prime divisor $p_{i}$ of $n$.

In this graph vertex 1 becomes an isolated vertex. When we enumerate the parameters total domination, connected domination, the contribution of this isolated vertex is nothing. Hence we take the divisors of $n$ excluding 1 as the vertex set of our Arithmetic $V_{n}$ graph and the adjacency is defined as above.

Clearly, $V_{n}$ graph is a connected graph. If $n$ is a prime, then $V_{n}$ graph consists of a single vertex. Hence it is connected. In other cases, by the definition of adjacency in $V_{n}$, there exist edges between prime number vertices and their prime power vertices and also to their prime product vertices. Therefore each vertex of $V_{n}$ is connected to some vertex in $V_{n}$.

\subsection{Direct Product Graphs}

Products of graphs occur naturally in discrete mathematics as tools in combinatorial constructions. They give rise to important classes of graphs and deep structural problems. In the beginning the emphasis was on the structure of finite and infinite products, but later it shifted to recognition algorithms for classes of isometric subgraphs of products of graphs, which are referred as product graphs. The methods recently developed for recognizing them have been extremely fruitful, have led to a better perception of the earlier work, and have helped to simplify many of the original proofs, both for finite and infinite graphs.

The structure and applicability of these products are quite interesting. For example, large networks such as the Internet graph, with several hundred million hosts, can be efficiently 
modeled by subgraphs of powers of small graphs with respect to the direct product. This is one of many examples of the dichotomy between the structure of products and that of their subgraphs. For a detailed description on product graphs refer $[3,4]$.

There are four standard products of graphs, namely those of the Cartesian, the strong, the direct and the lexicographic product.

In this paper, we consider direct product graphs of Cayley graphs with Arithmetic graphs and present Matching dominating set of these graphs.

In the literature, the direct product is also called as the tensor product, categorical product, cardinal product, relational product, Kronecker product, weak direct product, or conjunction.

Let $G_{1}$ and $G_{2}$ be two simple graphs with their vertex sets as $V_{1}=\left\{u_{1}, u_{2}, \ldots, u_{l}\right\}$ and $V_{2}=\left\{v_{1}, v_{2}, \ldots, v_{m}\right\}$ respectively. Then the direct product of these two graphs denoted by $G_{1} \times G_{2}$ is defined to be a graph with vertex set $V_{1} \times V_{2}$, where $V_{1} \times V_{2}$ is the Cartesian product of the sets $V_{1}$ and $V_{2}$ such that any two distinct vertices $\left(u_{1}, v_{1}\right)$ and $\left(u_{2}, v_{2}\right)$ of $G_{1} \times G_{2}$ are adjacent if $u_{1} u_{2}$ is an edge of $G_{1}$ and $v_{1} v_{2}$ is an edge of $G_{2}$.

The cross symbol $\times$, shows visually the two edges resulting from the direct product of two edges.

Now we consider the direct product graph of Euler totient Cayley graphs with Arithmetic $V_{n}$ graph. The properties and some dominating parameters of these graphs are presented respectively in $[5,6,7,8,9]$.

We state the following theorem without proof and the proof can be found in [7].

Theorem 1.3.1: Suppose $n$ is neither a prime nor $2 p$. Let $n=p_{1}^{\alpha_{1}} p_{2}^{\alpha_{2}} \ldots \ldots . . p_{k}^{\alpha_{k}}, \quad$ where $p_{1}, p_{2}, \quad \ldots p_{k}$ are primes and $\alpha_{1}, \alpha_{2}, \ldots . \alpha_{k}$ are integers $\geq 1$. Then the domination number of $G\left(Z_{n}, \varphi\right)$ is given by $\gamma\left(G\left(Z_{n}, \varphi\right)\right)=$ $\lambda+1$, where $\lambda$ is the length of the longest stretch of consecutive integers in $V$, each of which shares a prime factor with $n$.

We state the following theorem without proof and the proof can be found in [9].

Theorem 1.3.2: If $n \neq p^{\alpha}, n \neq 2 p$ and $n=p_{1}^{\alpha_{1}} p_{2}^{\alpha_{2}} \ldots \ldots . p_{k}^{\alpha_{k}}$ where $\alpha_{i} \geq 1$, then the domination number of $G_{1} \times G_{2}$ is given by

$\gamma\left(G_{1} \times G_{2}\right)=(\lambda+1) k$, where $\lambda$ is the length of the longest stretch of consecutive integers in $V_{1}$ of $G_{1}$ each of which shares a prime factor with $n$ and $k$ is the core of $n$.

\subsection{Matching Dominating Set}

A matching in a graph $G(V, E)$ is a subset $M$ of edges of $E$ such that no two edges in $M$ are adjacent. A matching $M$ in $G$ is called a perfect matching if every vertex of $G$ is incident with some edge in $M$.

A dominating set $D$ of $G$ is said to be a matching dominating set if the induced subgraph $\langle D\rangle$ admits a perfect matching.
The cardinality of the smallest matching dominating set is called matching domination number and is denoted by $\gamma_{m}$.

The matching domination number of Euler totient Cayley graph is presented in [5] and we state the theorem without proof and the proof can be found in [5].

Theorem 1.4.1: Let $n$ be neither a prime nor $2 p$ and $n=$ $p_{1}^{\alpha_{1}} p_{2}^{\alpha_{2}} \ldots \ldots . p_{k}^{\alpha_{k}}$, where $p_{1}, p_{2}, \ldots p_{k}$ are primes and $\alpha_{1}, \alpha_{2}, \ldots . \alpha_{k}$ are integers $\geq 1$. Then the matching domination number of $G\left(Z_{n}, \varphi\right)$ is given by

$$
\gamma_{m}\left(G\left(Z_{n}, \varphi\right)\right)= \begin{cases}\lambda+1 & \text { if } \lambda \text { is odd } \\ \lambda+2 & \text { if } \lambda \text { is even }\end{cases}
$$

where $\lambda$ is the length of the longest stretch of consecutive integers in $V$ each of which shares a prime factor with $n$.

\section{RESULTS}

\subsection{Matching Dominating Sets in Arithmetic $V_{n}$ Graph}

We now present the matching domination number of Arithmetic $V_{n}$ graph.

Theorem 2.1.1: Suppose $n=p_{1}^{\alpha_{1}} p_{2}^{\alpha_{2}} \ldots \ldots . . p_{k}^{\alpha_{k}}$, where $\alpha_{i} \geq 1$. Then the matching domination number of $G\left(V_{n}\right)$ is given by $\gamma_{m}\left(V_{n}\right)=\left\{\begin{array}{cl}k, & \text { if } \mathrm{k} \text { is even } \\ k+1, & \text { if } \mathrm{k} \text { is odd }\end{array}\right.$

where $k$ is the core of $n$.

Proof: Let $n=p_{1}^{\alpha_{1}} p_{2}^{\alpha_{2}} \ldots \ldots . . p_{k}^{\alpha_{k}}$, where $\alpha_{i} \geq 1$.

Case 1: Suppose $k$ is an even number.

Let $\quad D_{m}=\left\{p_{1}, p_{1} p_{2}, p_{3}, p_{3} p_{4}, \ldots \ldots \ldots ., p_{k-1}, p_{k-1} p_{k}\right\}$ and let $S=V-D_{m}$. All the vertices $u \in S$ for which $\operatorname{GCD}\left(u, p_{i}\right)=p_{i}$ for $i=1,3, \ldots, k-1$ are adjacent with the vertex $p_{i}$ in $D_{m}$. All the vertices $v \in S$ for which $G C D\left(v, p_{i} \cdot p_{i+1}\right)=p_{i+1}$ for $i=1,3, \ldots, k-1$ are adjacent with the vertex $p_{i} . p_{i+1}$. Since every vertex in $S$ has atleast one prime factor viz., $p_{1}, p_{2}, \ldots ., p_{k}$ (as they are the divisors of $n$ ) every vertex in $S$ is adjacent to atleast one vertex in $D_{m}$. Thus $D_{m}$ becomes a dominating set of $G\left(V_{n}\right)$.

Also the induced subgraph $\left\langle D_{m}\right\rangle$ admits a perfect matching with the pairs of vertices given by $\left\{p_{1}, p_{1} p_{2} ; p_{3}\right.$, $\left.p_{3} p_{4} ; \ldots \ldots \ldots ; p_{k-1}, p_{k-1} p_{k}\right\}$ as follows.

Any pair of vertices say, $p_{i}, p_{i} p_{i+1}$ in $D_{m}$ are adjacent since $\operatorname{GCD}\left(p_{i}, p_{i} p_{i+1}\right)=p_{i}$.

Also there is no edge between the vertices $p_{i} p_{i+1}$ and $p_{i+2}$ as $\operatorname{GCD}\left(p_{i} p_{i+1}, p_{i+2}\right) \neq p_{i}$ for any $i$. Further by the selection of pairs of vertices in $D_{m}$, no two pairs of vertices say, $p_{i}, p_{i} p_{i+1}$ and $p_{i+2}, p_{i+2} p_{i+3}$ are adjacent.

Thus $\left\langle D_{m}\right\rangle$ admits a perfect matching. Hence $D_{m}$ becomes a matching dominating set of $G\left(V_{n}\right)$. 
We now claim that $D_{m}$ is minimal. Suppose we remove any vertex $p_{i}$ from $D_{m}$. Then the paired vertex of $p_{i}$, viz., $p_{i} p_{i+1}$ is not adjacent to any other vertices of $D_{m}$ and becomes isolated in $\left\langle D_{m}\right\rangle$. Similar is the case if we delete any vertex $p_{i} p_{i+1}$ from $D_{m}$.

Again if we remove two vertices say $p_{j}$ and $p_{j} p_{j+1}$ from $D_{m}$ then an edge is removed in $\left\langle D_{m}\right\rangle$. But this does not make $D_{m}$ a dominating set.

Thus $D_{m}$ becomes minimal. If we construct matching dominating set in any other manner, then clearly it will be of larger size than $D_{m}$, or same size of $D_{m}$ because of properties of prime numbers.

Hence $\gamma_{m}\left(G\left(V_{n}\right)\right)=\left|D_{m}\right|=k$.

Case 2: Let $k$ be an odd number. Similar to case 1, it can be easily seen that $D_{m}^{\prime}=$

$$
\begin{cases}\left\{p_{1}, p_{1} p_{2}, p_{3}, \quad\right. & p_{3} p_{4}, \ldots \ldots \ldots, p_{k-2}, \\ & \left.p_{k-2} p_{k-1}, p_{k}, p_{k} p_{1}\right\}\end{cases}
$$

is a minimum matching dominating set where the induced subgraph $\left\langle D_{m}^{\prime}\right\rangle$ admits a perfect matching given by

$\left\{p_{1}, p_{1} p_{2} ; p_{3}, p_{3} p_{4} ; \ldots, p_{k-2}, p_{k-2} p_{k-1} ; p_{k}, p_{k} p_{1}\right\}$

Hence $\gamma_{m}\left(G\left(V_{n}\right)\right)=\left|D_{m}^{\prime}\right|=k+1$.

\subsection{Matching Dominating Sets in Direct Product Graph}

Theorem 2.2.1: If $n=p_{1}^{\alpha_{1}} p_{2}^{\alpha_{2}} \ldots \ldots . . p_{k}^{\alpha_{k}}, \quad$ where $\quad \alpha_{i} \geq 1$, then the matching domination number of $G_{1} \times G_{2}$ is given by

$$
\begin{gathered}
\gamma_{m}\left(G_{1} \times G_{2}\right)= \\
\left\{\begin{array}{cc}
(\lambda+1) \cdot k, & \text { if } \mathrm{k} \text { is even } \\
(\lambda+1) \cdot(k+1), & \text { if } \mathrm{k} \text { is odd }
\end{array}\right.
\end{gathered}
$$

where $\lambda$ is the length of the longest stretch of consecutive integers in $V_{1}$ of $G_{1}$ each of which shares a prime factor with $n$ and $k$ is the core of $n$.

Proof: Suppose $n=p_{1}^{\alpha_{1}} p_{2}^{\alpha_{2}} \ldots \ldots . p_{k}^{\alpha_{k}}$, where $\alpha_{i} \geq 1$. Consider the graph $G_{1} \times G_{2}$. By Theorem 1.3.2, we have $\gamma\left(G_{1} \times G_{2}\right)=(\lambda+1) \cdot k$ where $\lambda$ is the length of the longest stretch of consecutive integers in $V_{1}$ of $G_{1}$ each of which shares a prime factor with $n$ and $k$ is the core of $n$. Hence $\gamma_{m}\left(G_{1} \times G_{2}\right) \geq(\lambda+1) \cdot k$.

We know that $\gamma\left(G_{1}\right)=\lambda+1$ (by Theorem 1.3.1) and let $D_{1}=\left\{u_{d_{1}}, \ldots, u_{d_{\lambda+1}}\right\} \quad$ be a dominating set of $G_{1}$ with minimum cardinality, where $u_{d_{1}}, u_{d_{2}}, \ldots \ldots ., u_{d_{\lambda+1}}$ are consecutive integers. By Theorem 2.1.1, we know that matching dominating set of $G_{2}$ is

$$
\gamma_{m}\left(G_{2}\right)=\left\{\begin{aligned}
k, & \text { if } \mathrm{k} \text { is even } \\
k+1, & \text { if } \mathrm{k} \text { is odd }
\end{aligned}\right.
$$

Hence two cases arise.

Case 1: Suppose $\mathrm{k}$ is an even number. Then by Theorem 2.1.1, it can be easily seen that

$D_{2}=\left\{p_{1}, p_{1} p_{2} ; p_{3}, p_{3} p_{4} ; \ldots \ldots \ldots ; p_{k-1}, p_{k-1} p_{k}\right\} \quad$ is $\quad$ a minimum matching dominating set of $G_{2}$, as the induced sub graph $\left\langle D_{2}\right\rangle$ admits a perfect matching by the pairs of vertices given by $\left\{\left(p_{i}, p_{i} p_{i+1}\right) / i=1,3,5, \ldots, k-1\right\}$.

Now to obtain matching dominating set of $G_{1} \times G_{2}$, we proceed as follows.

$$
\text { Let } \begin{aligned}
D= & D_{1} \times D_{2} \\
= & \left\{u_{d_{1}}, u_{d_{2}}, \ldots \ldots ., u_{d_{\lambda+1}}\right\} \times \\
& \quad\left\{p_{1}, p_{1} p_{2} ; p_{3}, p_{3} p_{4} ; \ldots \ldots \ldots ; p_{k-1}, p_{k-1} p_{k}\right\} .
\end{aligned}
$$

Let $(u, v)$ be any vertex in $\langle V-D\rangle$ in $G_{1} \times G_{2}$. Then $u \in G_{1}$ is adjacent to $u_{d_{i}}$ for some $i$, where $1 \leq i \leq \lambda+$ 1 , as $D_{1}$ is a dominating set of $G_{1}$. Vertex $v \in G_{2}$ is adjacent to any of $p_{j}$ or $p_{j} p_{j+1}$, for $j=1,3,5, \ldots, k-1$ as $D_{2}$ is a dominating set of $G_{2}$.

Hence every vertex $(u, v)$ of $\langle V-D\rangle$ is adjacent to at least one vertex $\left(u_{d_{i}}, p_{j}\right)$ or $\left(u_{d_{i}}, p_{j} p_{j+1}\right)$ in $D$. This implies that $D$ is a dominating set of $G_{1} \times G_{2}$.

We now prove that $D$ is a matching dominating set of $G_{1} \times G_{2}$. Again two subcases arise.

Subcase 1: Suppose $\lambda$ is an even number. Then the induced subgraph $\langle D\rangle$ admits a perfect matching with the pairs of vertices given by

$$
\begin{aligned}
& \left\{\left\langle\left(u_{d_{i}}, p_{j}\right),\left(u_{d_{i+1}}, p_{j}, p_{j+1}\right)\right\rangle\right\} \cup \\
& \left\{\left\langle\left(u_{d_{\lambda+1}}, p_{j}\right),\left(u_{d_{1}}, p_{j} . p_{j+1}\right)\right\rangle\right\} \text { for } i=1,2,3, \ldots \ldots, \lambda \\
& \text { and } j=1,3,5, \ldots ., k-1 .
\end{aligned}
$$

For $i=1,2$ and $j=1,\left(u_{d_{1}}, p_{1}\right),\left(u_{d_{2}}, p_{1} \cdot p_{2}\right)$ and $\left(u_{d_{2}}, p_{1}\right),\left(u_{d_{3}}, p_{1}, p_{2}\right)$ are two pairs of vertices in $D$. Since $u_{d_{1}}, u_{d_{2}}$ and $u_{d_{3}}$ are all distinct and $p_{1} \neq p_{1} . p_{2}$, no two vertices among these four vertices are equal.

Further $u_{d_{1}}, u_{d_{2}}$ and $u_{d_{2}}, u_{d_{3}}$ are adjacent vertices because $\operatorname{GCD}\left(u_{d_{1}}-u_{d_{2}}, n\right)=1$ and $\operatorname{GCD}\left(u_{d_{2}}-u_{d_{3}}, n\right)=1$. Also $p_{1}, p_{1} \cdot p_{2}$ are adjacent vertices since GCD $\left(p_{1}, p_{1} . p_{2}\right)=p_{1}$. Therefore there exists edges between the pair of vertices $\left(u_{d_{1}}, p_{1}\right),\left(u_{d_{2}}, p_{1} . p_{2}\right) \quad$ and $\left(u_{d_{2}}, p_{1}\right),\left(u_{d_{3}}, p_{1}, p_{2}\right)$. This is true for all pairs of other vertices as these four vertices are arbitrary. Thus $\langle D\rangle$ admits a perfect matching and hence $\langle D\rangle$ becomes a matching dominating set of $G_{1} \times G_{2}$, with cardinality $(\lambda+1) k$. Then it follows by equation (1) that $\gamma_{m}\left(G_{1} \times G_{2}\right)=(\lambda+1) \cdot k$ 
Subcase 2: Suppose $\lambda$ is an odd number. Then the induced subgraph $\langle D\rangle$ admits a perfect matching with the pairs of vertices as follows.

$\left\{\left\langle\left(u_{d_{i}}, p_{j}\right),\left(u_{d_{i+1}}, p_{j} \cdot p_{j+1}\right)\right\rangle\right\} \cup$

$\left\{\left\langle\left(u_{d_{i+1}}, p_{j}\right),\left(u_{d_{i}}, p_{j} . p_{j+1}\right)\right\rangle\right\}$ for $\quad i=1,3,5 \ldots \ldots, \lambda$ and $j=1,3,5, \ldots, k-1$.

Consider any two pairs of vertices in $D$. Let them be $\left(u_{d_{1}}, p_{1}\right),\left(u_{d_{2}}, p_{1}, p_{2}\right)$ and $\left(u_{d_{2}}, p_{1}\right),\left(u_{d_{1}}, p_{1} . p_{2}\right)$.

Since $u_{d_{1}}, u_{d_{2}}$ are consecutive integers, GCD $\left(u_{d_{1}}-\right.$ $u d 2, n=1$ and GCD $p 1, p 1 . p 2=p 1$. Hence there exist edges between the pairs of vertices $\left(u_{d_{1}}, p_{1}\right)$, $\left(u_{d_{2}}, p_{1} . p_{2}\right)$ and $\left(u_{d_{2}}, p_{1}\right),\left(u_{d_{1}}, p_{1} . p_{2}\right)$.

Since $\quad u_{d_{1}} \neq u_{d_{2}}$ and $p_{1} \neq p_{1}$. $p_{2}$, no two vertices among these four are equal. This is true for all pairs of other vertices as these four vertices are arbitrary. Thus $\langle D\rangle$ admits a perfect matching and hence $\langle D\rangle$ becomes a matching dominating set of $G_{1} \times G_{2}$, with cardinality $(\lambda+1) k$. Then it follows by equation (1) that $\gamma_{m}\left(G_{1} \times G_{2}\right)=(\lambda+1) \cdot k$

Case 2: Suppose $k$ is an odd number. Then by Theorem 2.1.1, we can see that $D_{2}^{\prime}=$

$\left\{p_{1}, p_{1} p_{2} ; p_{3}, p_{3} p_{4} ; \ldots \ldots \ldots . . ; p_{k-2}, p_{k-2} p_{k-1} ; p_{k}, p_{k} p_{1}\right\}$ is a matching dominating set of $G_{2}$, with minimum cardinality $k+1$. Proceeding in the same way as in case 1 , we can prove that

$D^{\prime}=D_{1} \times D_{2}^{\prime}=\left\{u_{d_{1}}, u_{d_{2}}, \ldots \ldots ., u_{d_{\lambda+1}}\right\} \times$

$\left\{p_{1}, p_{1} p_{2} ; p_{3}, p_{3} p_{4} ; \ldots \ldots \ldots . . p_{k-2}, p_{k-2} p_{k-1} ; p_{k}, p_{k} p_{1}\right\}$

is a dominating set with minimum cardinality. Further the induced sub graph $\left\langle D^{\prime}\right\rangle$ admits a perfect matching as shown in the following two subcases.

Subcase 3: Suppose $\lambda$ is an even number, then

$$
\begin{aligned}
& \left\{\left\langle\left(u_{d_{i}}, p_{j}\right),\left(u_{d_{i+1}}, p_{j}, p_{j+1}\right)\right\rangle\right\} \\
& \cup\left\{\left\langle\left(u_{d_{i}}, p_{k}\right),\left(u_{d_{i+1}}, p_{k}, p_{1}\right)\right\rangle\right\} \\
& \cup\left\{\left\langle\left(u_{d_{\lambda+1}}, p_{j}\right),\left(u_{d_{1}}, p_{j} \cdot p_{j+1}\right)\right\rangle\right\} \\
& \cup\left\{\left\langle\left(u_{d_{\lambda+1}}, p_{k}\right),\left(u_{d_{1}}, p_{k} \cdot p_{1}\right)\right\rangle\right\}
\end{aligned}
$$

for $i=1,2, \ldots \ldots, \lambda$ and $j=1,3,5, \ldots, k-2$ gives a perfect matching.

For $i=1$ and $j=1$, consider two pairs of vertices $\left(u_{d_{1}}, p_{1}\right),\left(u_{d_{2}}, p_{1} . p_{2}\right)$ and $\left(u_{d_{1}}, p_{k}\right),\left(u_{d_{2}}, p_{k} . p_{1}\right)$. Since $u_{d_{1}} \neq u_{d_{2}}$ and $p_{1}, p_{1} . p_{2}, p_{k}, p_{k} . p_{1}$, are all distinct, it follows that no two vertices among these four vertices are equal. Also $\operatorname{GCD}\left(p_{1}, p_{1} . p_{2}\right)=p_{1}=\operatorname{GCD}\left(p_{k}, p_{k} . p_{1}\right)$ and GCD $\left(u_{d_{1}}-u_{d_{2}}, n\right)=1$ it follows that there exist edges between the pairs of vertices $\left(u_{d_{1}}, p_{1}\right),\left(u_{d_{2}}, p_{1} . p_{2}\right)$ and $\left(u_{d_{1}}, p_{k}\right),\left(u_{d_{2}}, p_{k} \cdot p_{1}\right)$. Thus $D^{\prime}$ becomes a matching dominating set with minimum cardinality $(\lambda+1) \cdot(k+1)$. Therefore $\gamma_{m}\left(G_{1} \times G_{2}\right)=(\lambda+1) \cdot(k+1)$.

Subcase 4: Suppose $\lambda$ is an odd number. Then the induced subgraph $\left\langle D^{\prime}\right\rangle$ admits a perfect matching given by the pairs of vertices

$$
\begin{aligned}
& \left\{\left\langle\left(u_{d_{i}}, p_{j}\right),\left(u_{d_{i+1}}, p_{j}, p_{j+1}\right)\right\rangle\right\} \cup \\
& \left\{\left\langle\left(u_{d_{i+1}}, p_{j}\right),\left(u_{d_{i}}, p_{j}, p_{j+1}\right)\right\rangle\right\} \cup \\
& \left\{\left\langle\left(u_{d_{i}}, p_{k}\right),\left(u_{d_{i+1}}, p_{k}, p_{1}\right)\right\rangle\right\} \cup \\
& \left\{\left\langle\left(u_{d_{i+1}}, p_{k}\right),\left(u_{d_{i}}, p_{k}, p_{1}\right)\right\rangle\right\}
\end{aligned}
$$

for $i=1,3, \ldots \ldots . \lambda$ and $j=1,3,5, \ldots, k-2$ as in subcase 3 . Thus $D^{\prime}$ becomes a matching dominating set with minimum cardinality $(\lambda+1) \cdot(k+1)$.

Therefore $\gamma_{m}\left(G_{1} \times G_{2}\right)=(\lambda+1) \cdot(k+1)$.

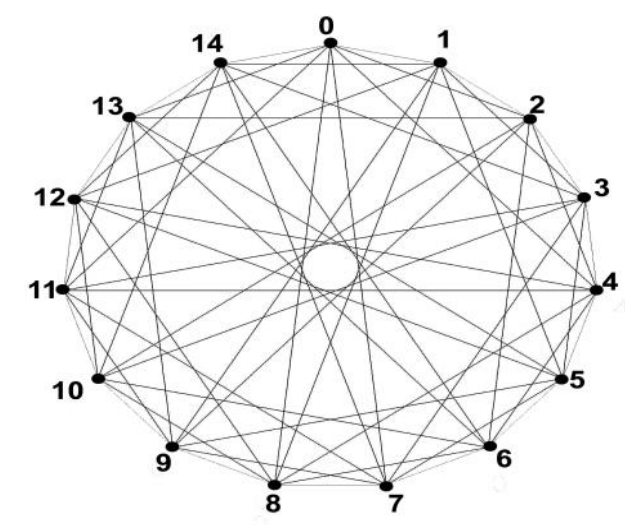

\section{ILLUSTRATIONS}

Let $n=15$

Fig. 1

$G_{1}=G\left(Z_{15}, \varphi\right)$ 


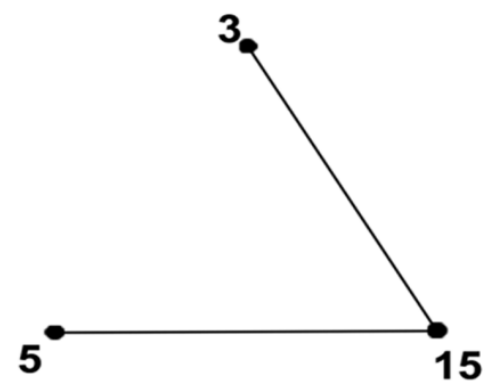

Fig. 2

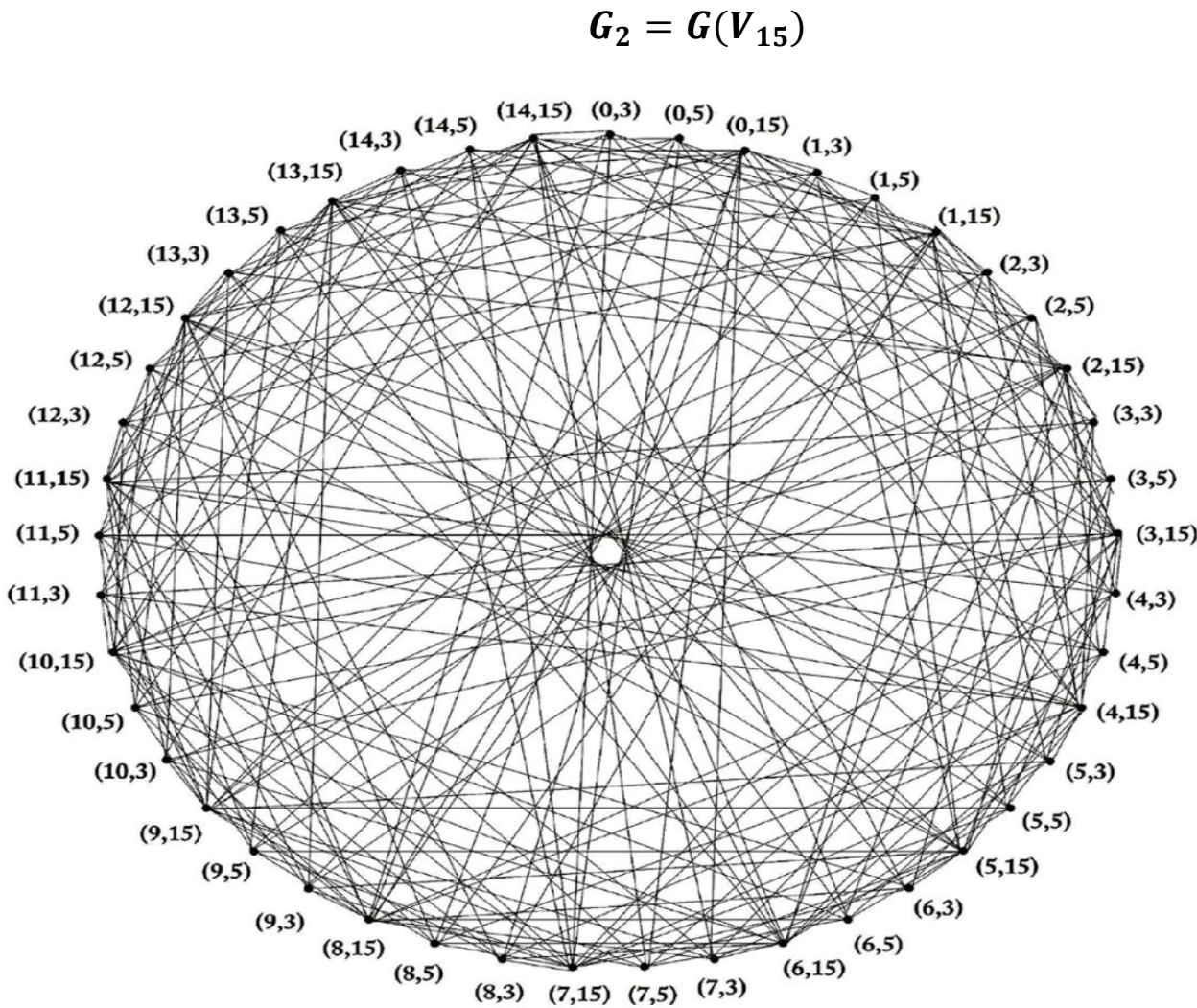

Fig. 3

$G_{1} \times G_{2}$

Table 1. Matching Dominations in Direct Product Graph $G_{1} \times G_{2}$

\begin{tabular}{|c|c|c|c|c|c|}
\hline $\begin{array}{c}\mathbf{n} \\
\text { Value }\end{array}$ & $\begin{array}{c}\text { Dominating } \\
\text { sets }\end{array}$ & $\boldsymbol{G}_{\mathbf{1}}=\mathbf{G}\left(\mathbf{Z}_{\mathbf{n}}, \boldsymbol{\varphi}\right)$ & $\boldsymbol{G}_{\mathbf{2}}=\boldsymbol{G}\left(\boldsymbol{V}_{\boldsymbol{n}}\right)$ & \multicolumn{1}{|c|}{$\boldsymbol{G}_{\mathbf{1}} \times \boldsymbol{G}_{\mathbf{2}}$} & $\begin{array}{c}\text { Domination } \\
\text { Number in } \\
\boldsymbol{G}_{\mathbf{1}} \times \boldsymbol{G}_{\mathbf{2}}\end{array}$ \\
\hline $\mathbf{n = 1 5}$ & $\begin{array}{c}\text { Minimum } \\
\text { Dominating set }\end{array}$ & $\{5,6,7\}$ & $\{15\}$ & $\{(5,3),(5,15),(6,3),(6,15),(7,3),(7,15)\}$ & $\gamma=6$ \\
\cline { 2 - 6 } & $\begin{array}{c}\text { Minimum } \\
\text { Matching } \\
\text { Dominating set }\end{array}$ & $\{5,6,7,8\}$ & $\{\langle 3,15\rangle\}$ & $\begin{array}{l}\{\langle(5,3),(6,15)\rangle ; \\
\langle(6,3),(7,15)\rangle ;\langle(7,3),(5,15)\rangle\}\end{array}$ & $\gamma_{m}=6$ \\
\hline
\end{tabular}




\section{REFERENCES}

[1] Madhavi, L. Studies on domination parameters and enumeration of cycles in some Arithmetic Graphs, $\mathrm{Ph}$. D. Thesis submitted to S.V.University, Tirupati, India, (2002).

[2] Vasumathi, N. Number theoretic graphs, Ph. D. Thesis submitted to S.V.University, Tirupati, India,(1994).

[3] Hammack, R. Imrich, W. and Klavzar, Handbook of product graphs, CRC Press, (2011).

[4] Weichsel, P.M. The Kronecker product of graphs, Proc. Amer. Math.Soc., 13, 47-52 (1962).

[5] Manjuri,M. and Maheswari, B.Matching dominating Sets of Euler Totient Cayley Graphs, International Journal of Computational Engineering Research (accepted).
[6] Uma Maheswari, S. and Maheswari, B. Domination parameters of Euler Totient Cayley graphs, Rev.Bull. Cal. Math.Soc.,19,(2),207-214(2011).

[7] Uma Maheswari, S. Some Studies on the Product Graphs of Euler Totient Cayley Graphs and Arithmetic Vn Graphs, Ph. D. Thesis submitted to S.P.Women's University, Tirupati, India, (2012).

[8] Uma Maheswari, S. and Maheswari, B. Some Domination parameters of Arithmetic Graph Vn, IOSR Journal of Mathematics, 6(2),14-18, (2012).

[9] Uma Maheswari, S, Maheswari, B.and Manjuri, M. Some Domination parameters of Direct Product Graphs of Cayley Graphs with Arithmetic Graphs, International Journal of Computer Applications (accepted) 